\title{
EXPERIENCIAS DE FORMACIÓN DOCENTE EN CONTEXTOS DE INSERCIÓN E INDUCCIÓN PROFESIONAL EN INSTITUCIONES BRASILEÑAS Y URUGUAYAS
}

\author{
Douglas da Silva Tinti \\ tinti@,ufop.edu.br \\ https://orcid.org/0000-0001-8332-5414 \\ Universidade Federal de Ouro Preto (UFOP) \\ Ouro Preto, Brasil. \\ Bárbara Cristina Moreira Sicardi Nakayama \\ barbara@ufscar.br \\ https://orcid.org/0000-0001-5097-459X \\ Universidade Federal de São Carlos, Campus Sorocaba (UFSCar)
}

Sorocaba, Brasil.

Recibido: 01/11/2020 Aceptado: 01/12/2020

\begin{abstract}
Resumen
El presente texto deviene de la experiencia de intercambio (práctica profesional) entre instituciones que forman a maestros y profesores en Brasil y en Uruguay y tiene como objetivo presentar elementos para que se comprenda cómo se caracterizan las prácticas de inserción e inducción profesional docente compartidas. Recurrimos, inicialmente, a algunos aportes teóricos para buscar conceptualizar la práctica de inserción e inducción profesional. Especialmente a partir de las definiciones sobre inicio de carrera e inserción profesional presentadas por Lima (1999), Vaillant \& Marcelo García (2012) y de inducción profesional fundamentadas en las contribuciones de Ferreira y Reali (2005) presentamos experiencias conducidas por cuatro instituciones formadoras que integran diferentes dimensiones de la práctica profesional, articulando cultura, currículo, investigación, política y demandas pedagógicas. Los resultados nos remiten al entendimiento de la colaboración y asociación entre institución formadora y escuela de Educación Básica y la relación inescindible entre enseñanza, investigación y extensión al configurarse como marcas y experiencias formadoras poderosas para iniciación a la docencia, inducción e inserción profesional.
\end{abstract}

Palabras clave: Formación docente. Inserción profesional. Experiencias curriculares. Asociación Universidad-Escuela. Práctica profesional.

\section{EXPERIÊNCIAS DE FORMÇÃO DOCENTE EM CONTEXTOS DE INSERÇÃO E INDUÇÃO PROFISSIONAL EM INSTITUIÇÕES BRASILEIRAS E URUGUAIAS}

\section{Resumo}

O presente texto decorre da experiência de intercambio (Passantía) entre instituições que formam professores no Brasil e no Uruguai e tem por objetivo apresentar elementos para que se compreenda como se caracterizam as práticas de inserção e indução profissional docente compartilhadas. Recorremos, inicialmente a alguns aportes teóricos para buscarmos conceituar a prática de inserção e indução profissional. Especialmente a partir das definições sobre início de carreira e inserção profissional apresentadas por Lima (1999), Vaillant \& Marcelo García (2012) e de indução profissional alicerçadas nas contribuições de em Ferreira e Reali (2005) apresentamos experiências conduzidas por quatro instituições formadoras que integram diferentes dimensões da prática profissional, articulando cultura, currículo, investigação, 
política e demandas pedagógicas. Os resultados nos remetem para o entendimento da colaboração e parceria entre instituição formadora e escola de Educação Básica e a indissociabilidade ensino pesquisa e extensão enquanto configuram-se como marcas e experiências formadoras potentes para a iniciação à docência, indução e inserção profissional.

Palavras-chave: Formação docente. Inserção profissional. Experiências curriculares. Parceria Universidade-Escola. Passantía.

\title{
TEACHER TRAINING EXPERIENCES IN CONTEXTS OF INDUCTION AND PROFESSIONAL INSERTION IN BRAZILIAN AND URUGUAYAN INSTITUTIONS
}

\begin{abstract}
This text stems from the experience of exchange (Internship) between institutions that train teachers in Brazil and Uruguay and aims to present elements so that it is possible to understand how the practices of shared professional teaching insertion and induction are characterized. We initially recurred to some theoretical contributions to try to conceptualize the practice of insertion and professional induction. Especially from the definitions of early career and professional insertion presented by Lima (1999), Vaillant \& Marcelo García (2012) and professional induction based on the contributions by Ferreira and Reali (2005), we present experiences conducted by four training institutions that integrate different dimensions of professional practice, articulating culture, curriculum, research, politics and pedagogical demands. The results lead us to the understanding of the collaboration and partnership between the training institution and the Basic Education school and the inseparability of teaching research and extension while being configured as powerful training marks and experiences for the initiation of teaching, induction and professional insertion.

Keywords: Teacher training. Professional insertion. Curricular experiences. University-School Partnership. Internship.
\end{abstract}

\section{Introducción}

A mediados de junio de 2017 fue abierto un Llamado Público para la realización de prácticas profesionales mediante el Proyecto Regional de Movilidad en Formación Docente Proyecto de Apoyo al Sector Educativo del MERCOSUR (PASEM) con financiamiento de la Unión Europea y de los países del MERCOSUR (Argentina, Brasil, Paraguay y Uruguay). Estaban habilitadas instituciones que ofrecen cursos de Formación Docente en los países miembros y asociados al MERCOSUR.

El objetivo del Llamado era el de apoyar la realización de prácticas profesionales entre docentes y otros actores educacionales, de dos o más instituciones de formación docente. Recepción y visita a otras instituciones. El valor financiado por el MERCOSUR era de USD 16.000 y las instituciones participantes deberían aportar USD 4.000 (20\%) como contrapartida. 
Considerando las exigencias del Edicto, elaboramos una propuesta que fue encaminada para su evaluación en octubre de 2017. A mediados de noviembre de 2017 tuvo lugar la divulgación de 10 proyectos aprobados y 10 en la condición de suplentes. Nuestra propuesta fue la segunda ubicada entre los proyectos aprobados.

Luego de un período considerable de espera de la liberación de los recursos, a mediados de diciembre de 2018 se nos notificó que deberíamos informar los datos bancarios para dar inicio al proyecto, que contó con la participación de 5 instituciones de formación de docentes y 10 investigadores/formadores que actúan en estas instituciones. Integran las instituciones participantes la Universidad Federal de Ouro Preto (UFOP), la Universidad Federal de São Carlos (UFSCar), la Universidad Cidade de São Paulo (UNICID), la Pontificia Universidad Católica de São Paulo (PUC/SP) y el Centro Regional de Formación de Profesores (CeRP) del Litoral en Salto/Uruguay.

Para este Proyecto de práctica profesional en términos de objetivo general se vislumbró desarrollar un estudio comparativo de políticas y prácticas institucionales de iniciación e inserción docente a partir de diferentes contextos formativos.

Para alcanzar este objetivo, inicialmente se realizó un mapeo de documentos que apoyan la formación de docentes en los países involucrados. A continuación, se buscó identificar proyectos y estudios acerca de la iniciación e inserción docente en las instituciones que integraron el proyecto. Como consecuencia de la definición de estas etapas fueron realizados dos viajes de práctica profesional, el primero de ellos a la UFOP y el segundo a la CeRP del Litoral con el propósito de compartir estudios, prácticas y experiencias de investigación sobre iniciación e inserción profesional docente en los dos países.

Pretendemos en este trabajo presentar elementos para que se comprenda cómo se caracterizan las prácticas de inducción e inserción profesional desarrolladas en Brasil y en Uruguay compartidas en el proyecto de práctica profesional de manera tal que se promuevan experiencias formadoras. En ese sentido, el texto a continuación se estructura con la perspectiva de presentar en un primer momento estudios para aclarar conceptos relacionados a la temática de la iniciación e inserción profesional docente y posteriormente discurrir sobre las prácticas y experiencias formadoras compartidas en ocasión de los viajes de práctica profesional. 


\section{Conceptos de Inserción e Inducción Profesional}

La constitución profesional es un movimiento complejo, extenso, personal y dependiente de muchos aspectos que impregnan no solamente la formación inicial y la continua, sino también la experiencia vivida como alumno, la trayectoria de vida del docente, el contexto político e histórico y, sobre todo, el contexto profesional en el cual el docente está inserto. El tornarse docente es un proceso extenso, desarrollado a partir de elementos teóricos, prácticos y personales, de la interiorización de conocimientos aprendidos en la formación inicial y en la práctica. Son los diversos eventos del ambiente escolar y de la trayectoria personal los que conducen a la constitución profesional. Asimismo, el reconocerse como docente es algo posterior al tornarse maestro o profesor, pues está relacionado con la imagen que el docente forma de sí, que no siempre se corresponde con la imagen que otros tienen de él.

Las primeras experiencias en el aula, en el actual sistema de licenciaturas brasileño, ocurren mientras el alumno está realizando los momentos de práctica (práctica profesional obligatoria y materias de práctica de enseñanza, en algunos casos), aunque ese contacto con la profesión puede ser limitado, ya que los alumnos no son los verdaderos responsables por el proceso educativo que integra su curso de formación. "En este momento, el contacto de los estudiantes con el campo profesional es exógeno, o sea, ellos todavía no son efectivamente profesionales" (Lima, 2006, p. 10). Aunque sea un momento formativo de extrema importancia para el futuro docente, es solamente en el ejercicio de la función que el profesional será capaz de comprender en profundidad las actividades por las cuales un docente es responsable.

La inserción en la carrera docente corresponde a los primeros años de ejercicio autónomo, en el cual el docente asume todas las atribuciones profesionales y desarrolla habilidades docentes exigidas, con la influencia de cuestiones personales y ambientales. Existe, sin embargo, un desacuerdo en cuanto a la duración de este período, aunque "la mayor parte de los investigadores de este dominio la hace corresponder a los tres primeros años de actividad docente (Pacheco \& Flores, 1999, p. 110). Vonk (1996, apud Vaillant \& Marcelo García, 2012, p. 130), por su parte, define "la inserción como la transición de docente en formación hasta llegar a ser un profesional autónomo. La inserción puede ser entendida mejor como una parte de un continuo en el proceso de desarrollo profesional de los docentes."

Considerándose la característica personal que influye en el desarrollo profesional docente, talvez el período de inserción docente tenga una duración variable en el sentido que 
Vonk propone, pues el alcance de la autonomía profesional depende de elementos escolares y personales. Algunas escuelas pueden promover un ambiente que ayude al maestro o profesor en ese proceso, mientras otras pueden dificultar el proceso. No obstante, en ambas situaciones, la inserción docente es un período de aprendizaje intenso para el docente iniciante.

La inducción, a su vez, es el seguimiento sistemático, intencional e institucionalizado que se hace del docente en proceso de inserción profesional o iniciación a la docencia. Según Ferreira y Reali (2005, p. 2):

Los programas de iniciación a la docencia, también denominados programas de inducción, son aquellos volcados a los docentes en sus primeras inserciones profesionales. [...] En general, estos programas ofrecen apoyo y orientación, con la perspectiva de promover el aprendizaje y el desarrollo de la base de conocimiento profesional y auxiliar en la socialización con la cultura escolar de esos profesionales.

Estudios dirigidos al mapeo de acciones destinadas a los docentes en inicio de carrera han demostrado, sobre todo a la luz de las contribuciones de Marcelo García (2009), que la organización de programas de inducción profesional puede ayudar al docente a enfrentar los desafíos para lidiar mejor con situaciones en el aula y en la escuela. Así, los programas de inducción deben ser comprendidos como una propuesta específica para una etapa que puede integrar tanto la formación inicial como la formación en servicio.

\section{Conceptos de experiencia y experiencia formadora}

Son varios los autores que nos ayudan a comprender el desarrollo profesional docente relacionado al concepto de experiencia. En nuestro caso será a partir de las contribuciones de Larrosa $(2002,2010)$ y Josso $(1988,2004)$ que presentaremos recursos para entender el concepto de experiencia formadora.

Encontramos en las contribuciones de Larrosa (2002) una primera indicación de que la experiencia integra mucho más que el tiempo de actuación en una cierta actividad, no es adquirida y sí vivida de modo que componga perspectivas, deje marcas y oriente elecciones. En tal dirección, Larrosa (2010) nos presenta una perspectiva del concepto relacionada al movimiento que el sujeto hace (o puede hacer) en la dirección de potenciar la vida, de la constitución de sí e indica que "la palabra experiencia nos ha servido y nos sirve para situarnos 
en un lugar o en el descubrimiento, a partir del cual no se dice: lo que no somos, lo que no queremos, sino que nos ha servido para afirmar nuestros deseos de vivir”. Así, el autor señala que "dejar que la experiencia tutele nuestra voz, nuestras producciones escritas, no es usar un instrumento, es situarse en el camino, esto es, en un espacio que está abierto a nosotros. Un espacio para el pensamiento, para el lenguaje, para la sensibilidad y para la acción, sobre todo, para la pasión" (p. 88).

Para este autor, el tiempo de nuestra vida está constituido por lo que sucede al pasar por las experiencias. "No es un acaecimiento entre una serie discreta de acaecimientos, sino un acaecimiento en el curso de la vida. Es en la historia de nuestras vidas que los acaecimientos asumen un orden y un sentido, así como también es en una trama que construimos nuestra propia continuidad o discontinuidad, a lo largo de los eventos de nuestra vida." (Larrosa, 2004, p. 17).

Por lo tanto, es necesario mirar, elaborar y expresar la experiencia, como narración, para que no se pierdan datos interesantes y sobresalientes sobre la trayectoria de formación, de las experiencias vividas que impelen los sujetos a las nuevas situaciones, en su propio hacer. Así, nos parece coherente traer al centro de estas reflexiones las contribuciones de Josso (2010) que nos revela el concepto de experiencia como algo que va reuniéndose y transformando el conocimiento en el transcurso de la vida, a partir del ejercicio de la escritura y la escucha de sí.

En ese sentido, Josso (2010) amplía la cuestión de la centralidad del sujeto en los procesos de formación, posibilitando la mirada desde la perspectiva de quien aprende, en interacciones con otras subjetividades. Para la autora:

Formarse es integrar en una práctica el saber hacer y los conocimientos, en la pluralidad de registros a que acabo de aludir. Aprender designa, más específicamente, el proprio proceso de integración. (Josso, 2010, p. 35)

Al entender que las prácticas de conocimiento puestas en juego, en un abordaje intersubjetivo, proporcionan el aprendizaje experiencial, Josso afirma que el sujeto, al aprender por la experiencia, será capaz de resolver problemas que él puede ignorar que tengan formulación y soluciones teóricas. Siendo así, el aprendizaje experiencial es utilizado en el sentido de resolver problemas, pero acompañado de una formulación teórica y/o simbolización (Josso, 2010, p. 36). 
Josso (2010, p. 56), al conceptualizar formación experiencial, pone en juego el paradigma individual y colectivo, pues el sujeto que aprende, en una actividad consciente de aprendizaje, tendrá la posibilidad de vivir interacciones consigo mismo, con los otros, con el medio en el que la actividad transcurre y en uno o varios registros. Siendo así, puede haber una integración de lo que es individual con lo que es colectivo, por medio de transformaciones psíquicas y comportamentales, acompañadas de sentimientos de diferentes naturalezas.

Para la autora, lo importante es el acceso y análisis de la experiencia como proceso de formación y las narrativas de formación, derivadas de las recordaciones, movilizan reflexiones de lo que los narradores consideran como experiencias significativas de sus aprendizajes y provocan diversas emociones que pueden revigorizar y fortalecer lo vivido, pudiendo transformarse en experiencia formadora. En ese sentido, no es lo que fue contado lo que es más importante, sino el sentimiento aflorado, el sentido que se da a esa memoria, cómo es resignificada. Es esa historia que tomamos para nosotros y nos reconocemos en ella. Y así, lo que da fuerza y significado a las experiencias vividas son las narrativas.

Entre esas experiencias narradas hay algunas situaciones dramáticas que son más intensas, que afloran mucho sentimiento, son muy fuertes, porque provocan cambios profundos, llevan a reflexionar y cambiar el recorrido de vida o de actuación. Esos momentos pueden ser considerados como lo que Josso (2010) llama "charnela". El término en francés se refiere a un tipo de bisagra y su movimiento recuerda el de abrir y cerrar, ir y volver. Pensando en esa analogía, la autora crea una alusión entre la articulación y los momentos de la vida, considerando que es en ese movimiento de idas y vueltas de las experiencias que la persona puede potenciar nuevos aprendizajes.

Aquí, a partir de las contribuciones teóricas de Larrosa y Josso, entendemos que las experiencias formadoras se consolidan en el ejercicio reflexivo de las vivencias. Se trata, por lo tanto, de un movimiento de potenciación del aprendizaje a partir de la identificación de las marcas que las vivencias promueven en nosotros y de la consecuente proposición de espacios de formación profesional que viabilicen este ejercicio. 
Una mirada sobre las prácticas de inducción e iniciación profesional articuladas al concepto de experiencia formadora: programas públicos, actividades curriculares y extensionistas en foco

A lo largo de las prácticas profesionales realizadas tanto en Brasil como en Uruguay, las instituciones participantes tuvieron la oportunidad de compartir contextos, prácticas y experiencias formadoras. Ese intercambio permitió mostrar puntos de aproximación y diferenciales de cada institución $\mathrm{y}$, consecuentemente, de los países participantes.

Los docentes vinculados a la UFSCar pudieron socializar el desarrollo del Programa Institucional de Beca de Iniciación a la Docencia (PIBID) y del Programa Residencia Pedagógica (PRP). El PIBID y el PRP -UFSCar entienden que la actuación del docente es situada, considerándose que esas acciones son individuales y al mismo tiempo colectivas. La enseñanza debe ser pensada con y no para las comunidades escolares. Tanto los futuros maestros y profesores, como aquellos que ya actúan en la Educación Básica son agentes de transformación e investigadores de su práctica. Así, el objetivo central del PIBID y el PRP UFSCar es articular la práctica de enseñanza y actividades relacionadas a esta con las cuestiones metodológicas. Las acciones desarrolladas con las escuelas son hechas a través de talleres, seminarios, proyectos interdisciplinarios temáticos, etc. Existe una preocupación por insertar a los estudiantes de licenciatura en proyectos que ya están siendo desarrollados en las Escuelas, de forma tal que se respeten las particularidades y singularidades de las mismas.

En el escenario nacional brasileño, el PIBID y el PRP se configuran como programas de inserción profesional, para cursantes de licenciatura, que ofrecen la oportunidad de experiencias formativas diferenciadas en el ambiente escolar - futuro locus de actuación profesional (Tinti, 2012). De ese modo, a partir de la socialización realizada por los docentes vinculados a la UFSCar, los docentes de la PUC-SP, UNICID y UFOP pudieron reflexionar sobre las diferentes contribuciones que ese Programa ha posibilitado para la formación de los futuros maestros y profesores.

Al referirnos a los cursos de licenciatura tenemos como presupuesto que uno de los elementos esenciales para la formación del licenciando es comprender que teoría y práctica se renuevan mutuamente. En ese sentido, concordamos con Sousa (2012) cuando afirma que el conocimiento de cómo saber enseñar se renueva y avanza a medida que la práctica, cotejada por la teoría, se revé y se innova al mismo tiempo que impulsa la teoría hacia las nuevas síntesis. Es en el interior de ese movimiento que, entendemos, se encuentran tanto el futuro docente como 
aquel que ya dicta clases desde algún tiempo, puesto que ambos pueden tornarse, mientras se forman, investigadores permanentes de la enseñanza.

Una práctica de integración curricular fue elegida por la docente de la UNICID para ser socializada. Es tradición del Curso de Pedagogía de la UNICID el desarrollo de Proyectos de Acción y de Intervención Integrados al Currículo, que - efectivamente - provoquen la inmersión de los futuros maestros y profesores en las causas profundas y urgentes de la educación en el mundo y en Brasil, así como también que ofrezcan oportunidades de reflexión, análisis, crítica y propuestas de soluciones para las problemáticas abordadas.

Dentro de este alcance, en 2018 - para ejemplificar con una aplicación más reciente - el Curso desarrolló el Proyecto Integrado “Amabilidad genera Amabilidad”, cuya constitución y desarrollo alcanzaron los contenidos actitudinales de la formación docente y abrieron una gama de posibilidades para que los futuros docentes lidien con situaciones volcadas a esa base de contenidos. La docente relató que se buscó, de manera amplia, abordar la temática de las relaciones éticas, humanas y profesionales en el área de la educación, a partir de contextos definidos, de fuentes seleccionadas y de materiales de apoyo coherentes con la propuesta.

En el ámbito de la UFOP, el docente que integró el proyecto optó por socializar una experiencia vinculada a la extensión universitaria, buscando mostrar las posibilidades formativas que pueden surgir de ese contexto. La propuesta de extensión socializada apuntaba a la constitución de un grupo de docentes que enseñan matemática, en diferentes momentos de la carrera docente, para la elaboración conjunta de una agenda de aprendizajes pautada en las investigaciones sobre la propria práctica. El proyecto contó con la participación de alumnos de los cursos de Pedagogía y Licenciatura en Matemática, además de un docente de la Educación Básica y el coordinador del proyecto. En ese sentido, el ambiente constituido posibilitó la interacción entre los miembros del grupo y la escuela participante.

El docente relató que el grupo que se constituyó para el desarrollo de la extensión universitaria optó por trabajar con los alumnos del $6^{\circ}$ grado de la Enseñanza Primaria de una Escuela Estadual, dado que esa era una demanda de la propria escuela.

Componiendo la pluralidad de posibilidades formativas, el Programa de Estudios de Posgrado en Educación: Formación de Formadores - FORMEP de la PUC/SP fue presentado como un Máster Profesional orientado hacia la formación del formador, corroborando la importancia del desarrollo de estudios dirigidos a ese profesional, destacando el objetivo de 
contribuir con los estudios que ya fueron realizados y mostrando aspectos identificados en tales estudios. De acuerdo con la Fundación CAPES (2019)

El Máster Profesional (MP) es una modalidad de Posgrado stricto sensu dirigida a la capacitación de profesionales, en las diversas áreas del conocimiento, mediante el estudio de técnicas, procesos, o temáticas que atiendan a alguna demanda del mercado de trabajo, debiendo además presentar [...] estructura curricular que enfatice la articulación entre conocimiento actualizado, dominio de la metodología pertinente y aplicación orientada al campo de actuación profesional específico; y [...] el trabajo final del curso debe estar siempre vinculado a problemas reales del área de actuación del profesional-alumno y de acuerdo con la naturaleza del área y la finalidad del curso.

Aquí, vale la pena mencionar el artículo 18 de la Decisión del CONSUN Nº 16/15 (PUC, 2015), que define a este formador como: [...] el profesional que lleva a cabo la mediación entre los profesionales de la educación, los alumnos y el conocimiento, con vistas a la cualificación de la acción educativa y sus implicaciones en el planeamiento, en la acción pedagógica y en la evaluación (PUC, 2015, p. 6).

A partir del habla de la docente que integra el proyecto de práctica profesional queda claro que la elección por invertir en la formación de un profesional en una perspectiva investigativa otorga al mismo la responsabilidad de actuar en diversas instancias y contextos, conforme las acepciones descritas por Vaillant (s/a) y Marcelo García (1999): formador como docente; como persona que forma a los docentes; como tutor de prácticas; como profesor mentor; como asesor de formación y también el formador en la educación no formal. Esta pluralidad de posiciones puede potenciar experiencias formadoras en la medida en que las mismas son también marcadas por el carácter académico.

En el contexto de las experiencias formadoras desarrolladas y vividas en la CeRP/Uruguay, se destaca el papel de la materia de Didáctica que posee las características de las Prácticas Profesionales Curriculares Supervisadas en Brasil.

A lo largo de 4 años de formación docente, los estudiantes cursan 4 materias de Didáctica. En el primer año, el enfoque es una introducción a la Didáctica (materia de naturaleza teórica). En el segundo y tercer año, los estudiantes cursan las materias de Didáctica I y II, siendo estas de naturaleza teórico-práctica. A lo largo de ellas, los futuros maestros y profesores son insertados en el contexto escolar, observan, planean y desarrollan algunas regencias. Hay un seguimiento de los docentes de la materia y de la escuela. Durante esa inserción, los 
formadores emiten algunos feedbacks con vistas a auxiliar a los futuros docentes en ese proceso de aprendizaje de la docencia.

Aunque en Uruguay la formación de docentes ocurra en nivel terciario, otro aspecto sobresaliente de esa práctica socializada se refiere al hecho que, en el $4^{\circ}$ año de la formación, los maestros y profesores en formación asumen un curso durante todo el año escolar. Es decir que quedan a su cargo el planeamiento, la conducción de las clases y los procesos evaluativos. Se trata de una atribución que es realizada por las Secretarías de Educación, siendo una actividad remunerada.

\section{Aspectos destacados finales: cuando la inducción se configura como experiencia formadora}

Al llegar este momento de síntesis y conclusión de texto, podemos ya tener más claridad acerca de cómo se caracterizan las prácticas de inserción e inducción profesional desarrolladas en Brasil y en Uruguay, compartidas en el proyecto de práctica profesional, de modo que promuevan experiencias formadoras.

En ambos contextos, el inicio en la profesión de docente es reconocido como un período de socialización con los nuevos colegas, estudiantes, comunidad, sistema educacional, red de enseñanza, normativas y directrices que comprenden el espacio educacional. Se cree que esta “entrada" en la profesión, o mismo, en otra red de enseñanza, sea privada o pública, presenta al docente nuevos desafíos, exigencias profesionales, modos de hacer y pensar la docencia, en virtud del nuevo contexto en que se adentra.

Observando individualmente las experiencias formadoras socializadas por las diferentes IES, notamos que el PIBID y el PRP de la UFSCar cargan en su esencia la posibilidad de viabilizar el encuentro y trabajo compartido entre maestros y profesores en ejercicio y futuros docentes. La práctica de integración curricular desarrollada en el curso de Pedagogía de la UNICID promueve la participación y el compromiso social con la lucha por mejores condiciones formativas y de trabajo, así como por derechos de ciudadanía y búsqueda de una sociedad más ética, justa e igualitaria. El proyecto de la UFOP encuentra sus bases en la relación

inescindible entre enseñanza, investigación y extensión, y ofrece la oportunidad de delineamiento compartido entre docentes en ejercicio y formación inicial de una agenda de aprendizajes pautada en las investigaciones sobre la propia práctica. El FORMEP apuesta a una 
formación investigativa de profesionales en ejercicio con la perspectiva de potenciar la esfera de la corresponsabilidad por el acogimiento y perfeccionamiento docente en la escuela básica.

Las experiencias desarrolladas por diferentes instituciones aquí relatadas integran múltiples dimensiones de la práctica profesional, articulando cultura, currículo, investigación, política y demandas pedagógicas. Se observa que, en todas las experiencias narradas, el principio de inducción concebido como estrategia de valorización del magisterio y mantenimiento en la carrera docente está presente. Sea a partir del trabajo articulado a programas de iniciación a la docencia, actividades curriculares, extensionistas, investigativas o de práctica profesional. Lo que queda latente en la manera de organizar las experiencias es el principio de inducción relacionado al movimiento de insertar el futuro docente en el contexto de la escuela en contacto y / o en asociación con profesionales en ejercicio marcado por procesos reflexivos.

Así, cuando caracterizamos las prácticas de inserción e inducción profesional docente a partir de las experiencias compartidas, identificamos que las mismas no se diferencian conceptualmente, pero sí en carácter contextual y didáctico. En Brasil, son movilizadas por las prácticas profesionales curriculares supervisadas y por dos programas públicos nacionales (PIBID y PRP) a la vez que en el contexto uruguayo el énfasis yace en una acción estructurada curricularmente (Materia de Didáctica) impulsando el docente en formación a asumir efectivamente un curso de alumnos por un año lectivo, siendo acompañado por los formadores. De este modo, comprendemos que la manera como las prácticas son conducidas es lo que las configura como experiencias formadoras que potencian los procesos de inducción.

Sin embargo, pautar estas prácticas de manera reflexiva viabiliza el entendimiento de la colaboración, la asociación entre institución formadora y escuela de educación Básica y la relación inescindible entre enseñanza, investigación y extensión como potencia formadora para la constitución e inserción profesional docente. Se trata, por lo tanto, de experiencias formadoras pues promueven la escucha de sí mismo, la reflexión y el aprendizaje compartidos y la participación en el contexto profesional.

\section{Referencias}

Brasil. Fundação CAPES (2014). Mestrado Profissional: o que é?. Recuperado de: https:/capes.gov.br/avaliacao/sobre-a-avaliacao/mestrado-profissional-o-que-e 
Ferreira, L. A.; Reali, A. M. de M. R. (2005). Aprendendo a ensinar e a ser professor: Contribuições e desafios de um programa de iniciação à docência para professores de Educação Física. In: Anais da 28 Reunião Anual da ANPED, Caxambu, 2005. p. 1-17. Recuperado de: www.anped.org. br/reunioes/28/inicio.htm

Lima, E. F. (1999) Sobrevivências: no início da docência. Brasília: Líber Livro Editora, 2006. In.: Pacheco, J. A.; Flores, M. A. (1999) Formação e Avaliação de Professores. Portugal: Porto Editora.

Josso, M. C. (1988). Da formação do sujeito ao sujeito da formação. In: NÓVOA, A. \& FINGER, M. O método (auto) biográfico e a formação. Lisboa: Ministério da Saúde.

Josso, M. C. (2004). Experiências de vida e formação. São Paulo: Cortez.

Larrosa, J. (2004) Notas sobre narrativa e identidade (a modo de presentación). In: Abrahão, M. H. M. B. (Org.). A aventura (auto)biográfica: teoria e empiria. Porto Alegre: EDIPUCRS.

Larrosa, J. (2010). Herido de realidad y en busca de realidad. Notas sobre los lenguajes de la experiencia. In: Contreras; J.; Pérez de Lara, N. F. (Comps). Investigar la experiência educativa. Madrid: Morata.

Marcelo García, C. (1999). Formação de professores. Para uma mudança educativa. Colecção Ciências da Educação Século XXI. Porto Editora, Portugal.

Marcelo García, C. (2009). Desenvolvimento Profissional: passado e futuro. sísifo-Revista das ciências da educação, n. 08, p. 7-22, jan./abr. 2009.

Pacheco, J. A.; Flores, M. A. (1999). Formação e Avaliação de professores. Porto: Ed. do Porto.

Pontifícia Universidade Católica de São Paulo. Mestrado Profissional em Educação: Formação de Formadores. Deliberação do CONSUN $n^{0}$ 16/2015. Recuperado de: https://www.pucsp.br/sites/default/files/download/posgraduacao/programas/educacaof ormacaoformadores/deliberacao_do_consun_no_16-2015.pdf_novo_0.pdf

Sousa, M. C. (2012). Formação docente nas licenciaturas da UFSCar: contribuições do PIBID. Espaço Plural, Ano XIII, No 26, $1^{\circ}$ Semestre 2012, p. 55- 69.

Tinti, D. S. (2012) PIBID: um estudo sobre suas contribuições para o processo formativo de alunos de Licenciatura em Matemática da PUC-SP. 148f. Dissertação (Mestrado em Educação Matemática) - Programa de Estudos Pós-Graduados em Educação Matemática. Pontifícia Universidade Católica de São Paulo. São Paulo.

Vaillant, D. (s/a). Formação de Formadores. [S.1.: s.n.] s/a, p. 275-301. Recuperado de: http://www.uel.br/eventos/enfopli/pages/arquivos/Formacao_de_Formadores.pdf

Vaillant, D.; Marcelo García, C. (2012). Ensinando a ensinar: quatro etapas de uma aprendizagem. Curitiba: UTFPR. 